\title{
Mining Surveillance Data: Does Radiation Treatment of Prostate Cancer Cause Rectal Cancer?
}

\author{
John W. Morgan*1, ${ }^{2}$, Brice Jabo¹, Mark E. Ghamsary ${ }^{1}$ and Kevork Kazanjian ${ }^{3}$ \\ 1'Dept Epidemiology, Biostatistics \& Population Medicine, Loma Linda University School of Public Health, Loma Linda, CA, USA; \\ ${ }^{2}$ Desert Sierra Cancer Surveillance Program, Region 5 of California Cancer Registry, Loma Linda, CA, USA; ${ }^{3}$ Dept of Surgery, \\ Division of Surgical Oncology, Loma Linda University School of Medicine, Loma Linda, CA, USA
}

\section{Objective}

We sought to assess whether external beam radiation (RAD) treatment of prostate cancer, that exposes the rectum to ionizing radiation, was followed by increased hazards for rectal cancer, relative to prostatectomy (SURG).

\section{Introduction}

Prostate cancer (PC) is the most common invasive cancer diagnosed among US men. The majority of PCs are organ-confined at diagnosis making them candidates for treatment using RAD, SURG, or other protocols. Several studies have provided preliminary evidence that radiation treatment of prostate cancer may increase subsequent rectal cancer risk (1-2). Data specifying type of RAD treatment of $\mathrm{PC}$ was not available for the study period.

\section{Methods}

We conducted record linkage for all 322,327 organ confined new prostate cancers and 53,204 new rectum and rectosigmoid junction (rectal) cancers among California males from 1988-2009, identifying men diagnosed with rectal cancer more than five years following treatment of organ-confined prostate cancer with RAD or SURG. Among the men treated with RAD vs SURG, the Cox proportional hazards ratio (HR) for subsequent rectal cancer was assessed. Demographic covariates included: race/ethnicity as Asian/Other (A-O), non-Hispanic black (NHB), Hispanic (Hisp), and non-Hispanic white (NHW), and socioeconomic status quintiles (1-5 Highest). Other covariates included age, as a continuous variable, and year of PC diagnosis.

\section{Results}

Among the 43,130 men having organ-confined prostate cancer that had been treated with RAD only, 166 were diagnosed with rectal cancer more than five years following PC treatment. Likewise, 69,104 men treated with SURG only, yielded 242 rectal cancer cases more than 5 years later. Following is the demographic factor adjusted hazards ratio (HR) for rectal cancer with $95 \%$ confidence intervals (CI) contrasting findings for the two PC treatment cohorts: Rectal cancer
$\mathrm{HR}_{\mathrm{RAD} / \mathrm{SURG}}=1.39 ; 95 \% \mathrm{CI}=1.12-1.74$. HR contrasts for demographic factors included age- $\left(\mathrm{HR}_{\mathrm{Age}}=1.02 ; 95 \% \mathrm{CI}=1.01-1.04\right)$, race/ethnicity- $\left(\mathrm{HR}_{\mathrm{A}-\mathrm{ONHW}}=1.10 ; 95 \% \mathrm{CI}=0.72-1.67, \mathrm{HR}_{\mathrm{NHBNHW}}=1.19 ; 95 \%\right.$ $\mathrm{CI}=0.82-1.74$ and $\left.\mathrm{HR}_{\mathrm{Hisp} / \mathrm{NHW}}=1.01 ; 95 \% \mathrm{CI}=0.72-1.43\right)$, and SEScontrasts $\left(\mathrm{HR}_{\mathrm{SES} 1 / \mathrm{SES} 5}=0.95 ; 95 \% \mathrm{CI}=0.65-1.39\right), \mathrm{HR}_{\mathrm{SES} 2 / \mathrm{SESS}}=1.20$; $95 \% \mathrm{CI}=0.89-1.62, \mathrm{HR}_{\mathrm{SES} 3 / \mathrm{SES} 5}=1.17 ; 95 \% \mathrm{CI}=0.88-1.55$, and $\left.\mathrm{HR}_{\mathrm{SES} 4 / \mathrm{SESS}}=1.14 ; 95 \% \mathrm{CI}=0.87-1.49\right)$. The $\mathrm{HR}$ for PC year of diagnosis $\left(\mathrm{HR}_{\text {Year }}=0.91 ; 95 \% \mathrm{CI}=0.89-0.94\right)$ a protective effect for more recent years.

\section{Conclusions}

These findings reveal increased hazards for rectal cancer among organ-confined prostate cancer patients treated with RAD, relative to patients treated with SURG, that are substantially independent of demographic covariates. Treatment of rectal cancer among these patients is further complicated because they are ineligible for radiation treatment of rectal cancer due to the high-dose pelvic radiation received during prostate cancer treatment. Further analyses that seek to distinguish roles of different dose and delivery methods for RAD are ongoing.

\section{Keywords}

radiation; cancer; rectal; prostate; surveilance

\section{References}

1. E Rapiti, G Fioretta, HM Verkooijen, R Zanetti, F Schmidlin, H Shubert, A Merglen, R Miralbell, C Bouchardy. Increased risk of colon cancer after external radiation therapy for prostate cancer. Int. J. Cancer: 123, 1141-1145 (2008).

2. NN Baxter, JE Tepper, SB Durham, DA. Rothenberger,BA Virnig. Increased Risk of Rectal Cancer After Prostate Radiation: A PopulationBased Study. Gastroenterology 2005;128:819-824.

*John W. Morgan

E-mail: john.w.morgan@att.net 